\title{
Development of Sugar Free and Fortified Chocolates with D-Optimal Design Approach
}

\author{
Aishwarya Paranjape, Sachin Sonawane, Sonal Patil* \\ School of Bioinformatics and Biotechnology, D Y Patil Deemed to be University, Navi Mumbai, India \\ E-mail:ppsonal@rediffmail.com (Corresponding author)
}

Received: 24 April 2021; Accepted: 13 May 2021; Available online: 10 June 2021

\begin{abstract}
The increasing numbers of overweight or chronically obese individuals have led the market to produce sweet snacks and desserts that are low in calories, low in fat or low in sugar. Sugar replacers have low calorific value and lower glycaemic index as compared to sucrose which not only helps in controlling weight but also is safe for diabetics. The study is aimed to develop a sugar free chocolate by using a combination of various zero calorie sweeteners i.e., stevia, isomalt and erythritol through response surface methodology (RSM). The final run optimized by RSM consists of $21.9 \mathrm{~g}$ cocoa butter, $5.1 \mathrm{~g}$ erythritol, $0.10 \mathrm{~g}$ stevia and $14.9 \mathrm{~g}$ isomalt. Organoleptic evaluation of modified chocolates was evaluated using 9 point hedonic scale for the parameters like, appearance, color, aroma, taste, texture, mouthfeel, aftertaste and overall acceptability. The overall acceptability of sugar free chocolates was found to be 8.9. Further, the fortification of chocolates was done with the help of vitamins and minerals rich fruit powders such as beetroot powder, jamun seed powder and pink pithaya powder (dragon fruit). Vitamins and minerals analysis was performed for sugar free chocolate fortified with beetroot. Shelf-life study of sugar free chocolate fortified with beetroot was carried out.
\end{abstract}

Keywords: Sugar free; Sweetener; Response surface methodology; Fortification.

\section{Introduction}

The word 'chocolate' is derived from Nahuatl word 'Xocolatl' meaning 'bitter water'. In its original embodiment in the Mayan and Aztec societies, it refers to as a hot and spiced beverage. Chocolate being a solid mixture, is composed of cocoa powder, cocoa butter, and certain type of sweeteners likes sugar. However, today's modern chocolates consist of milk solids, added flavours, as well as modifiers, and the preservatives. The quantity of sugar, mass, or the cocoa butter used in the chocolates strongly varies from manufacturer to manufacturer, and this remains a basic trade secret. Through the process of manufacturing of chocolate product, the characteristics can vary. Hence, accurate standardization is required to make standard and quality cocoa products [1]. Polyphenols, called as phytonutrients are the main components found in plants that are believed to promote and improve the human health. In the cacao plant, polyphenols work as a defense mechanism [2]. Flavonoids are believed to be responsible for chocolate's health benefits through their antioxidant and anti-inflammatory functions in the body. Flavonoids are seen to activate antioxidant enzymes. These important functions depict chocolate as having significant medicinal uses [2]. People with diabetes always face difficulty in balancing their blood sugar levels. Hence, by limiting their sugar consumption by replacing it with artificial sweeteners, they can enjoy all varieties of food. Various reports are available stating about the ability of some sugar substitutes to release energy slowly, allowing stable blood sugar levels over the time. Persons with reactive hypoglycemia produce an excess of insulin when glucose is quickly absorbed into the bloodstream. This results into the fall of blood glucose levels below the advisable amount for physiological functions. Hence, need to select artificial sweeteners as a best alternative sugar for diabetic persons. There is an increase in interest in sugar reducing chocolates and the preferred natural sweetener substitutes. The main sugar substitutes which are used in this study are Stevia, Erythritol and Isomalt. As per the "National Centre for Complementary and Integrative Health Trusted Source", foods high in antioxidants, like beets, are most beneficial in preventing ailment. Beets contains antioxidants which is nearly 1.7millimoles/3.5 ounces that includes a group of antioxidants also called as betalains, the main component responsible for its reddish color. The beetroots also contain other composites that can help suppress inflammation [3].

Demand for food products with less sugar content is increasing the presence of natural or artificial sweeteners plays an important role because sugar replacers have low calorific value and lower glycaemic index as compared to sucrose and are safe for diabetics. Thus, the study aimed to develop a sugar free chocolate by using a 
combination of various zero calorie sweeteners i.e., Stevia, Isomalt and Erythritol through response surface methodology (RSM) approach.

\section{Materials and methods}

\subsection{Materials}

Hershey's cocoa powder (100\% natural unsweetened), pure milk powder and cocoa butter from Morde was purchased from local market in Kharghar. Beetroot powder of Nature’s Gift, Jamun seed powder and pink Pithaya Powder (dragon fruit) of Urban Platter was purchased through Amazon.

Sweetners like Stevia (Aoxing Stevia, Shandong, China), Erythritol (Baolingbao Biology Co.Ltd, china), Monk fruit sweetener of Urban Platter was purchased through Amazon. Samples of isomalt were gifted by BENEOPalatinit GmbH.

Chemicals like 1, 1-Diphenyl-2-picrylhydrazyl (DPPH), methanol, ethanol, acetone, Gallic acid, HCl, Lascorbic, Petroleum ether (PET), Folin-ciocalteau reagent, quercetin were from SRL Company. And other chemicals like sodium chloride, magnesium chloride, iron chloride, nitric acid, potassium thiocyanate and other reagents used in this study were of analytical grade.

Media used in this study were Nutrient broth, agar agar type 1 from SRL company.

Equipments like oven, tray dryer (Classic Scientific, Mumbai), muffle furnace (Hally Instruments), double beam UV-VIS Spectrophotometer (Spectroscan UV 2600, Chemito), high performance liquid chromatography (thermofisher scientific), gas chromatography, hunter lab colorimeter, incubator, grinder was used to perform the different tests.

\subsection{Methods}

\subsubsection{Preparation of basic chocolates}

Cocoa butter was melted over a medium heat by using double boiler method. In a bowl all the dry ingredients in i.e., cocoa powder, milk powder, sugar was mixed together which was followed by grinding to avoid grainy texture of chocolates. The melted cocoa butter was removed from heat followed by the addition of the powdered mixture of dry ingredients to form a smooth paste. The paste was then poured into the mold kept into refrigerator for 25-30 minutes and samples were removed out of the mold when set.

\subsubsection{One factor optimization of cocoa powder, sugar and cocoa butter}

One factor optimization of proportions of ingredients used for the preparation of chocolates were carried out with the trial-and-error method where concentration of one ingredient varied keeping all other ingredients at a constant proportion. The varying concentration of ingredients is as shown in table 1.

Table 1. Varying concentration used for preparation of chocolates

\begin{tabular}{lll}
\hline Cocoa powder $(\%)$ & Sugar $(\%)$ & Cocoa butter $(\%)$ \\
\hline 10 & 11.25 & 16.5 \\
12 & 15 & 22 \\
15 & 18.75 & 27.5 \\
20 & 22.5 & 33 \\
25 & 26.5 & \\
\hline
\end{tabular}

\subsubsection{Determination of sweetness intensity}

The sweeteners like sugar, stevia, isomalt, erythritol \& monk fruit were individually added in drinking water in concentration of $0.001 \%, 0.01 \%, 0.10 \%, 1 \%$ and $10 \%$. For each sweetener different solution of sweetener and water were prepared recorded.

\subsubsection{Sensory evaluation}

Sensory quality evaluation of samples was performed with the help of 9-point hedonic scale. A panel of 10 semi-trained healthy individuals falling between the age group of 18 to 35 years was involved in the sensory analysis. Samples were served in plastic cups at room temperature $\left(30^{\circ} \mathrm{C}\right)$. Evaluation was based on color, aroma, mouth feel, taste, texture, aftertaste \& overall acceptability. Each panelist scores were reflected on a 9 point hedonic scale where 1 was ‘dislike extremely’ and 9 was ‘like extremely’.

\subsubsection{Fortification of sugar free chocolates}

Fortification of sugar free chocolates was done with the help of beetroot powder, jamun seed powder and pink pithaya powder incorporation one at a time. Chocolates were prepared with varying concentrations of each powder 
i.e., $4 \%$ and $7 \%$ with and without masking its taste with $0.79 \%$ of coffee wherever required.

\subsubsection{Proximate analysis}

The proximate analysis of the samples included determination of moisture content (\%), ash content (\%), fat content (\%), protein content (\%) and carbohydrate content (\%) as per AOAC (1990) procedures. The total calorific value of the samples was also calculated according to the calorific value of the individual elements present in the chocolates [4].

\subsubsection{Experimental design}

D-optimal design of response surface methodology was employed for obtaining experimental design for the optimization of sweeteners incorporation during the preparation of sugar free chocolates. Design Expert version 7.0 software was used. As replacement of sugar with sweeteners is also responsible for reduction in the bulk of chocolate; cocoa butter was selected as one of the factors for the design along with erythritol, stevia and isomalt. Sensory attributes such as taste, mouthfeel, texture and aftertaste were the studied responses. Twenty chocolate formulations were processed by using these three basic sweeteners. Overall acceptability (OA) was measured in terms of sensory attributes. The regions of maximum OA were identified and marked on counterplots for sensory response. The experimental design set up is elaborated in table 2.

\subsubsection{ANOVA statistics of model terms}

All the variables and responses were applied to the standard quadratic model by performing the analysis of variance (ANOVA). Further, the results obtained were analyzed to decide the lack of fit. The lack of fit test measures the failure of a model to represent the experimental data at the point excluded in the regression. While the goodness-of-fit of the model was identified by the coefficient of determination, $\left(\mathrm{R}^{2}\right)$ and it should be at least around $80 \%$.

\section{Results and discussion}

\subsection{One factor optimization of chocolate}

It was found that, cocoa powder, sugar and cocoa butter in the amount of $15 \mathrm{gm}, 22.5 \mathrm{gm}$ and 16.5 gm were found to produce chocolates with maximum acceptability scores. Hence, the same proportion was used for further studies.

\subsection{Sweetness intensity of sweeteners}

The sweeteners used to test their sweetness intensity were Sugar, Erythritol, Monk Fruit, Stevia, Isomalt. The concentrations for which the tests performed were $0.001 \%, 0.01 \%, 0.10 \%, 1 \%$ and $10 \%$ respectively. Isomalt showed sweetness intensity as high as 10 at the proportion of 10\%, while Erythritol showed sweetness intensity 2 at $1 \%$ of its concentration. Monk fruit showed the first sweetness intensity at $0.10 \%$ concentration. When Stevia was used at $0.001 \%$ concentration it showed the sweetness intensity as 5 . This means it is sweetest among all the sweeteners used. The sweetness intensity was found to be stevia $>$ monk fruit $>$ erythritol $>$ isomalt.

\subsection{Response surface methodology (RSM) results}

Table 2 shows the data of varying responses with respect to variations in the combinations of sweeteners and cocoa butter. Changes in the combination as well as concentration of these factors have found to impact sensory attributes of sugar free chocolates

As can be seen from figure 1, mouthfeel of the sugar free chocolates was influenced by varying concentrations of sweeteners and cocoa butter. Increase in erythritol reduced mouthfeel whereas increase in the concentration of cocoa butter brought out an increase in the scores of mouthfeel for sugar free chocolates owing to the presence of free fatty acids in cocoa butter as well as it's microcrystalline structure. The cooling effect of erythritol when combined with fats may be responsible for reduction in mouthfeel scores [5].

Cocoa butter concentration was found to be increasing the acceptability scores for taste of sugar free chocolates whereas acceptability scores for taste of sugarfree chocolates were decreased with increase in erythritol concentration owing to the cooling effect of erythritol. Increase in isomalt also led to an increase in taste score of sugar free chocolates.

Texture of sugar free chocolates was also influenced by variation in it's ingredients. Increase in cocoa butter improved texture whereas increase in erythritol and isomalt negatively hampered the texture owing to their property to form waxy structure when mixed fatty acids in cocoa butter [5].

Aftertaste was not significantly affected with the variation in these ingredients. 
Table 2. experimental design for optimization experiments for developing sugar free chocolate using response surface methodology (ingredients in gram)

\begin{tabular}{lllllllll}
\hline Run & A: Cocoa butter & B: Erythritol & C: Stevia & D: Isomalt & Taste & Mouthfeel & Texture & Aftertaste \\
\hline 1 & 18.5 & 8.4 & 0.15 & 15 & 7.3 & 6.7 & 7.1 & 7.4 \\
2 & 19.4 & 15 & 0.20 & 7.4 & 7.7 & 8 & 7.6 & 7.7 \\
3 & 21.9 & 14.8 & 0.20 & 5.1 & 8.1 & 7.5 & 8.2 & 7.9 \\
4 & 18.5 & 11.3 & 0.20 & 12.1 & 8.1 & 7.8 & 7.7 & 7.7 \\
5 & 15 & 14.7 & 0.15 & 12.2 & 6.9 & 6.6 & 6.9 & 8 \\
6 & 15 & 11.9 & 0.10 & 15 & 7 & 7.1 & 7.4 & 2.2 \\
7 & 21.7 & 7.1 & 0.20 & 15 & 8.1 & 8.1 & 8 & 7.7 \\
8 & 21.9 & 5.1 & 0.10 & 12.9 & 8 & 8.2 & 8.2 & 8 \\
9 & 15 & 11.9 & 0.10 & 15.0 & 6.5 & 6.3 & 6.6 & 8.2 \\
10 & 21.9 & 7.1 & 0.10 & 12.9 & 7.7 & 7.25 & 7.5 & 8.3 \\
11 & 21.9 & 8.3 & 0.20 & 11.6 & 8 & 7.6 & 7.6 & 7.6 \\
12 & 21.7 & 5.1 & 0.20 & 15 & 8.6 & 8.4 & 8.5 & 7.8 \\
13 & 16.4 & 12.6 & 0.20 & 12.8 & 6.8 & 6.6 & 6.5 & 7 \\
14 & 19.8 & 9.2 & 0.19 & 12.9 & 8 & 7.9 & 7.6 & 7.8 \\
15 & 21.9 & 15 & 0.10 & 5 & 7.9 & 7.5 & 7.5 & 8 \\
16 & 15.6 & 11.2 & 0.20 & 15 & 7.1 & 7 & 7 & 7.1 \\
17 & 17 & 15 & 0.20 & 9.8 & 7 & 6.7 & 7.1 & 7 \\
18 & 15 & 14.7 & 0.15 & 12.2 & 7.7 & 6.6 & 6.5 & 7.6 \\
19 & 20.9 & 12.1 & 0.20 & 8.8 & 7.7 & 7.2 & 7.4 & 7.4 \\
20 & 21.9 & 15 & 0.10 & 5.0 & 8 & 8 & 7.4 & 7.2 \\
\hline
\end{tabular}

\subsection{Optimization of constrains and validation of model terms}

Table 3 gives us the range for each of the parameter involved in the RSM analysis. Comparing the values obtained from the desired model with this ideal range, it was observed that all the parameters, cocoa butter, Erythritol, Stevia and Isomalt were in the desired range. Also the sensory parameters like taste, mouthfeel, texture and after taste were observed to be maximum.

Table 3. Optimization of Constrains and Validation of Model Terms

\begin{tabular}{lllllll}
\hline Name & Goal & Lower Limit & Upper Limit & Lower Weight & Upper Weight & Importance \\
\hline Cocoa butter & Is in range & 15 & 21.9 & 1 & 1 & 3 \\
Erythritol & Is in range & 5.09521 & 15 & 1 & 1 & 3 \\
Stevia & Is in range & 0.1 & 0.2 & 1 & 1 & 3 \\
Isomalt & Is in range & 5.01564 & 15 & 1 & 1 & 3 \\
Taste & Maximize & 6.5 & 8.6 & 1 & 1 & 3 \\
Mouthfeel & Maximize & 6.3 & 8.4 & 1 & 1 & 3 \\
Texture & Maximize & 6.5 & 8.5 & 1 & 1 & 3 \\
Aftertaste & Maximize & 7 & 8.3 & 1 & 1 & 3 \\
\hline
\end{tabular}

\subsection{ANOVA statistics of model terms}

The results obtained from the ANOVA analysis are shown in Table 4 Comparison of the F and p-values indicate that the quadratic model of the sugar free chocolate development is statistically significant. The lack of fit for each response is insignificant. The $\mathrm{R}^{2}$ values for the taste, after taste, texture and mouthfeel are range from $58 \%$ to $70 \%$ respectively.

Table 4. ANOVA Statistics of Model

\begin{tabular}{lllll}
\hline Model terms & After taste & Texture & Mouth feel & Taste \\
\hline Prob $>$ F & 0.0022 & 0.0005 & 0.0011 & 0.0002 \\
Std.Dev & 0.28 & 0.35 & 0.43 & 0.33 \\
Mean & 7.73 & 7.71 & 7.35 & 7.61 \\
R-squared & 0.581 & 0.6631 & 0.6228 & 0.6991 \\
Lack of fit & Insignificant & Insignificant-ant & Insignificant & Insignificant \\
\hline
\end{tabular}

\subsection{Solutions given by RSM}


Based on the above results of sensory analysis of the chocolate formulated using different sweeteners, Sugar, cocoa butter, Erythritol, Stevia, Isomalt, a very good desired results were obtained. Response surface methodology was well suited for evaluating the variables on sensory characteristics of chocolate. The optimized formulation contained $21.9 \%$ cocoa butter, $5.1 \%$ Erythritol or $0.10 \%$ of Stevia or $14.9 \%$ of Isomalt to establish best possible desirability of 0.771 .

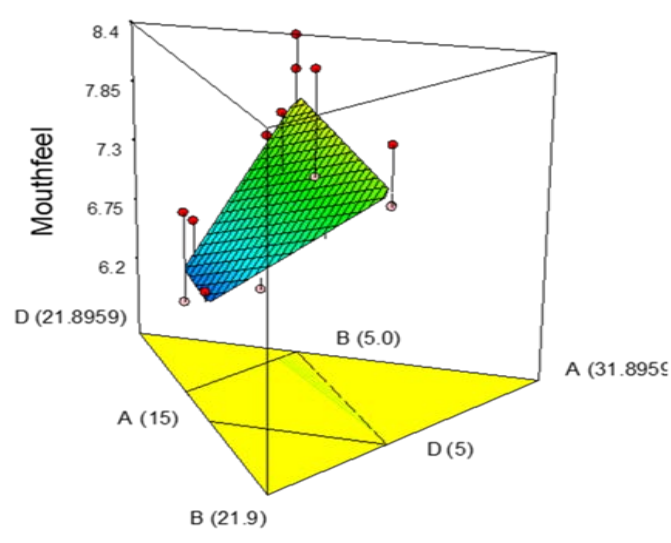

(A)

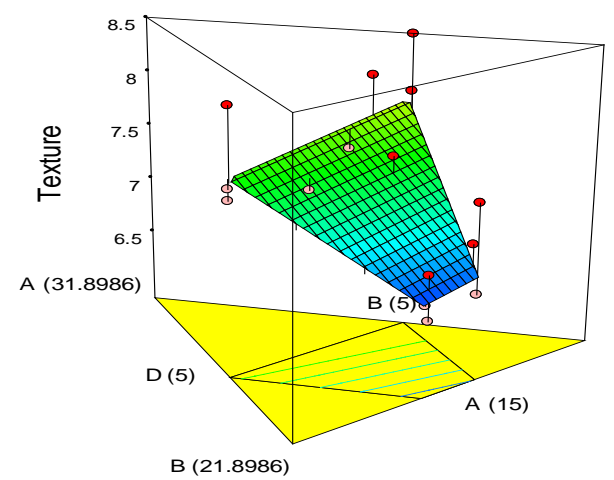

(C)

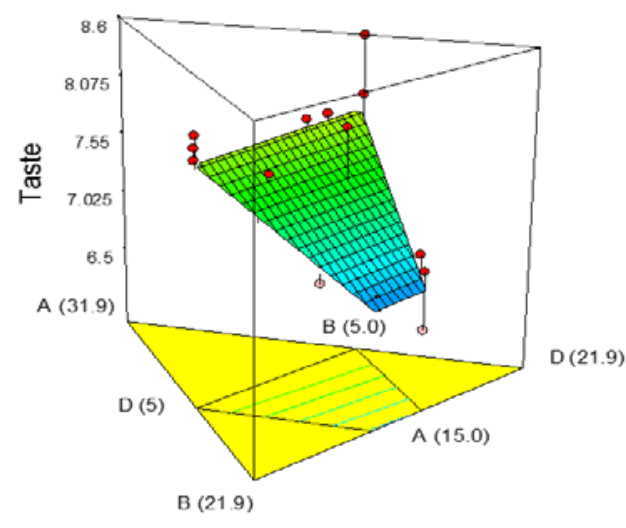

(B)

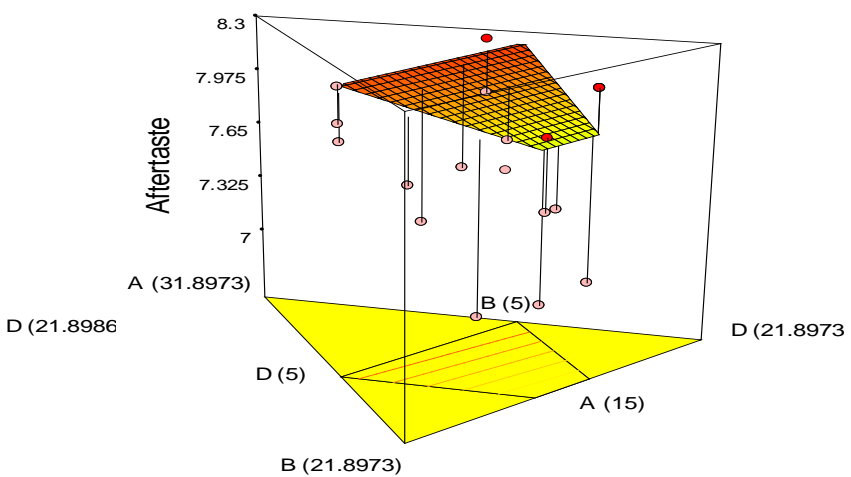

(D)

Figure 1. Effect of components on sensory quality A) mouthfeel B) taste C) texture D) aftertaste

\subsection{Sugar free chocolates fortified with powders of beetroot, Jamun and Dragan fruit}

The results showed that the sugar free chocolate prepared with $4 \%$ of beetroot, jamun and dragon fruit powder was most optimized without masking and when masked with $1 \%$ coffee $4 \%$ of beetroot, jamun and dragon fruit powder showed optimized results.

\subsection{Proximate analysis of sugar free chocolates fortified with beetroot, jamun and dragon fruit powder}

Results showed that chocolates fortified using beetroot powder resulted in increased protein and fibre content in chocolates than by other powders. Results showed that chocolates fortified using beetroot powder resulted in increased protein and fibre content in chocolates in accordance with the findings of [6-8]. Also the level of vitamin $\mathrm{D}$ content is observed higher in beet fortified chocolate as compared to the sugar free chocolate sample. According to a research, it is observed that beetroot contains some amount of Vitamin D [9]. Sugar Free chocolates presented larger amounts of fats, 33.6 \%g/g: $11.84 \mathrm{~g}$ of SFA (saturated fatty acid), $12.54 \mathrm{~g}$ of MUFA (monounsaturated fatty acid), and $9.2 \mathrm{~g}$ of PUFA (polyunsaturated fatty acid) per $100 \mathrm{~g}$ of chocolate. Sugar free chocolates fortified using beetroot powder had the lowest amounts of fats and hence it can be recommended as the healthiest option than regular chocolate, followed by chocolates fortified using Jamun Powder [10]. The higher moisture content value in the beetroot sample might be due to the high fiber content in beetroot. 
More hydroxyl groups of cellulose in fiber were able to bind with free water molecules through hydrogen bonding and thus resulting in greater water holding capacity [11]. We observed that the sugar free chocolates fortified with Jamun Powder presented nearest Ash Level value to the controlled sample value.

\subsection{Mineral analysis of sugar free chocolates}

The amount of Magnesium in the sugar free sample $(237.9 \mathrm{mg} / 100)$ is observed to be less than its value in chocolate fortified with beetroot powder (318.3 mg/100), The iron had appreciable values of $2.9 \mathrm{mg} / 100 \mathrm{~g}$ in sugar free chocolate then $1.3 \mathrm{mg} / 100 \mathrm{~g}$ in beetroot fortified chocolate.

\section{Conclusion}

The sensory analysis was performed for all 20 types of chocolates and the final run generated by RSM had $21.9 \mathrm{~g}$ cocoa butter, $5.1 \mathrm{~g}$ erythritol, $0.10 \mathrm{~g}$ stevia and $14.9 \mathrm{~g}$ isomalt with optimum sensory attributes. The sugar free chocolates prepared with $4 \%$ of fruit powder and $1 \%$ of coffee (to mask the after taste) was optimized.

Future study can be in development of sugar free candy using monk fruit sweetener, development of sugar free chocolate drink fortified with mango leaves or in development of sugar free confectionery using Tagatose fortified with combination of beetroot, dragon fruit and jamun powder.

\section{References}

[1] Nair KPP. Cocoa (Theobroma cacao L.). The Agronomy and Economy of Important Tree Crops of the Developing World. Elsevier. 2010. p.131-180.

[2] Heim KE, Tagliaferro AR, Bobilva DJ. Flavonoid antioxidants: Chemistry, metabolism and structure-activity relationships. Journal of Nutritional Biochemistry. 2002;13:572-584.

[3] Adrienne Santos-Longhurst, 2019, Beetroot and diabetes: Should you eat them?. https://www.healthline. com/health/diabetes/beetroot-diabetes.

[4] AOAC. Official methods of analysis, $18^{\text {th }}$ ed. Association of Official Analytical Chemists, AOAC International, Gaithersburg, MD, USA. 2005.

[5] Sheng-Dun L. Ching-Ching L, Jeng-Leun M, Li-Yun L, Shiow-Ying C. Effect of erythritol on quality characteristics of reduced-calorie Danish cookies. Journal of Food Quality. 2010; 33: 14-26.

[6] Uthumporn U, Woo WL, Tajul AY, Fazilah A. Physico-chemical and nutritional evaluation of cookies with different levels of eggplant flour substitution. Journal of Food. 2015;13 (2): 220-226.

[7] Pinki and Pratima A. Sensory and nutritional evaluation of value added cakes formulated by incorporating beetroot powder. International Journal of Food and Nutritional Sciences. 2014; 3(6):145-148.

[8] Jenkins DJ, Kendall CW, Marchie A, Faulkner DA, Wong JM, De Souza R, Connelly P. Effects of a dietary portfolio of cholesterol-lowering foods vs lovastatin on serum lipids and creactive protein. JAMA. 2003; 290:502-510.

[9] Amount of Vitamin D in Beets, http://www.dietandfitnesstoday.com/vitamin-d-in-beets.php, https://en.wikipedia.org/wiki/Beetroot

[10] Ranawana V, Moynihan E, Campbell F, Duthie G, Raikos V. Beetroot improves oxidative stability and functional properties of processed foods: singular and combined effects with chocolate. Journal of Food Science and Technology. 2018; 55(7): 2401-2409. doi:10.1007/s13197-018-3157-3

[11] Rosell CM, Rojas JC, Benedito DB, Nobhan GP and Truswell ASC. Influence of hydrocolloids on dough rheology and bread quality. Food Hydrocolloids. 2001; 15:75-81.

(C) 2021 by the author(s). This work is licensed under a Creative Commons Attribution 4.0 International License (http://creativecommons.org/licenses/by/4.0/). Authors retain copyright of their work, with first publication rights granted to Tech Reviews Ltd. 